\title{
Chapter 9 \\ Delayed Luminescence in Relation \\ to the Germination and Vigour of Coffee \\ Seeds: Initial Series with $C$. Arabica \\ Samples
}

\author{
Cristiano M. Gallep, Lilian Padilha, Mirian P. Maluf \\ and Sttela D.V.F. da Rosa
}

\begin{abstract}
Ultra-weak delayed luminescence measurements on coffee seeds were run for samples submitted to different post-harvest treatments or in seed lots with different vigour levels obtained by accelerated aging, and both were induced to germinate afterwards. Parameters of hyperbolic decay fitting were correlated to the correspondent germination vigour, with a linear relation found for the initial value as well as for the decay velocity. These preliminary data point to further, broader validation for this non-invasive, non-destructive test for seed's viability analyses.
\end{abstract}

\subsection{Introduction}

The coffee seed normally presents high germination potential just after appropriate harvest and desiccation. However, it also loses its physiological quality very rapidly under common storing conditions. Therefore, it is not possible to have viable seeds, i.e. able to germinate, for more than some months [1].

Some techniques may improve seed's viability on long term, by improving storing conditions [2, 3], or controlling the re-hydration process [4] or even inducing low-temperature hibernation [5].

Although some progress was achieved in this sense the usual way for checking seeds' viability and vigour is to allow them to germinate, i.e. losing so the hibernation condition. Furthermore, the results from germination tests are only available in 30 days after imbibition. So, new strategies able to save time are crucial in

C.M. Gallep $(\bowtie)$

Applied Photonics Lab, School of Technology, University of Campinas, Limeira, SP, Brazil e-mail: gallep@ft.unicamp.br

L. Padilha · M.P. Maluf · S.D.V.F. da Rosa

Brazilian Agricultural Research Corporation, Embrapa/Coffee Unit, Brasilia, DF, Brazil

e-mail: lilian.padilha@embrapa.br

(C) Springer International Publishing Switzerland 2016 
determining the proper actions for the conservation and commercialization of coffee seeds. In order to distinguish between viable and not viable seeds, and so enable an optimization of seed's storage conditions, a quick and non-destructive method is demanded, as well for other types of sensitive seeds.

The biophotonic phenomena, i.e. the ultra-weak delayed luminescence and spontaneous emission found in living organisms, with detected intensity of 10-1000 photons $/ \mathrm{cm}^{2} \mathrm{~s}$, has been studied by many multi-disciplinary groups all over the world, in a broad variety of themes [6]. This peculiar luminescence holds much longer than the usual bio-fluorescence, and is found far from normal thermal black-body emission, covering the entire visible spectrum and the near IR and UV [7].

Correlations between the ultra-weak delayed luminescence (DL) behaviour and the germination capacity were found already for barley [8], soybean [9], rice [10] and wheat [11] seeds. A first, small trial with coffee seeds were performed by the first author ten years ago at IIB facilities (Neuss, Germany), with some indicative results of good correlation between the DL parameters and the germination capacity of tested seeds [12]. At that time, just three different lots were tested, with medium and high germination capacity.

Here, preliminary series of ultra-weak DL measurements of coffee seeds are presented in relation to their germination rate and total seedling elongation measured in the hypocotyl-radicle axis. Seeds submitted to different post-harvest treatments and seed lots with different vigour levels were tested for delayed luminescence, and induced to germinate afterwards.

The germination performance was established after 15 and 30 days and correlated to DL parameters. Good correlation was found between the germination vigour and the initial intensity and the decay velocity of the correspondent DL curves.

\subsection{Materials and Methods}

Initially, the DL of nine groups of coffee seeds (Coffea Arabica), harvested in June/2011 and treated in different conditions, were analysed in terms of hyperbolic decay in May/2013, and further induced to germinate in controlled conditions.

These seeds were harvest, processed and stored in controlled conditions at Federal University of Lavras (UFLA, MG, Brazil). The different seed groups ('A' series) are presented at Table 9.1-if mucilage is removed mechanically or by fermentation, if drying was done in drying machine, in shadow or under sun-light, and the moisture content achieved after drying (12\% or $35 \%)$.

In a second series of tests, ' $\mathrm{B}$ ', nine seed lots from Seed Analysis Laboratory-UFLA were submitted to accelerated ageing test $\left(42{ }^{\circ} \mathrm{C}, 100 \%\right.$ relative humidity, for $48 \mathrm{~h}$ ) to produce lots with different vigour levels, as presented at Table 9.2. Germination tests were run before and after the seed aging to evaluate the seed performance. DL tests were run in June/2013. 
Table 9.1 Coffee seed groups 'A'-different post-harvest treatments

\begin{tabular}{l|l|l}
\hline Group & Post-harvest treatment & Moisture content (\%) \\
\hline A7 & mechanical removal of mucilage, mechanical dryer & 12 \\
\hline A9 & mechanical removal of mucilage, dried in shadow & 12 \\
\hline A11 & mechanical removal of mucilage, sun-dried & 12 \\
\hline A13 & removal of mucilage by fermentation, mechanical dryer & 12 \\
\hline A14 & removal of mucilage by fermentation, mechanical dryer & 35 \\
\hline A15 & removal of mucilage by fermentation, dried in shadow & 12 \\
\hline A16 & removal of mucilage by fermentation, dried in shadow & 35 \\
\hline A18 & removal of mucilage by fermentation, sun-dried & 12 \\
\hline
\end{tabular}

Table 9.2 Coffee seed groups ' $\mathrm{B}$ '- accelerated aging test

\begin{tabular}{l|l|l|l}
\hline \multirow{2}{*}{ Sample } & \multicolumn{2}{l|}{$\begin{array}{l}\text { Germination rate } \\
(\%)\end{array}$} & \multirow{2}{*}{ Rating (vigour) } \\
\cline { 2 - 3 } & Initial & Aged $^{\mathrm{a}}$ & \\
\hline 1 & 74 & 41 & Low \\
\hline 2 & 86 & 83 & High \\
\hline 3 & 85 & 78 & High \\
\hline 4 & 78 & 77 & Medium \\
\hline 5 & 78 & 80 & High \\
\hline 6 & 71 & 75 & Medium \\
\hline 7 & 76 & 75 & Medium \\
\hline 9 & 82 & 80 & High \\
\hline
\end{tabular}

${ }^{\mathrm{a}}$ Accelerated aging test: seeds under $48{ }^{\circ} \mathrm{C} / 100 \%$ R.H. for $48 \mathrm{~h}$

Random samples of $50 \mathrm{~g}$ were taken from each seed group for the ultra-weak DL measurements and stored in dark to avoid artefacts. The experimental setup for DL tests are shown at Fig. 9.1; it is a dark chamber with photon-count module (photomultiplier tube + electronics) and a fiber optic ring connect to external light source (halogen lamp) by a fiber cable and electrical-mechanical shutter, all automatic controlled by software; it includes also temperature control for samples using fluid flow [13].

Each group of seeds was arranged in the chamber in order to complete the sample holder, which was stabilized in temperature $\left(T=21 \pm 1{ }^{\circ} \mathrm{C}\right)$ to avoid seed stress. The DL measurements used photon-count mode in $100 \mu$ s time-windows for 20 thousand points (total $=2 \mathrm{~s}$ ) and were taken after twenty-second exposure to white light (160 lux), and repeated sequentially ten times for each sample. A delay of $370 \mathrm{~ms}$ occurs between the end of excitation and photon-count start due technical limitation. 


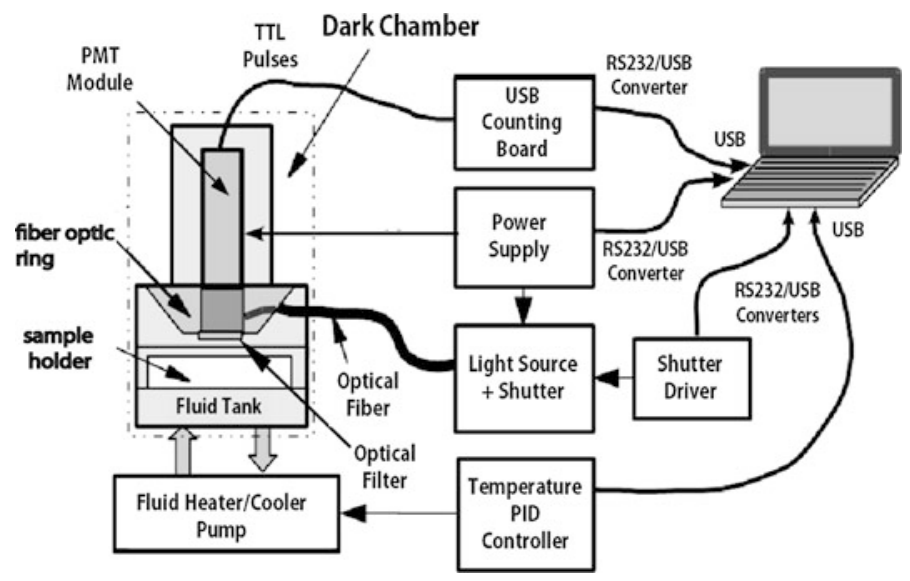

Fig. 9.1 Setup for DL measurements in biosamples-(top) chamber, illumination and controls schematics; (bottom) picture of prototype

The 10-repetition DL data was averaged and the curve was fitted by generic hyperbolic-like decay, formulated by:

$$
a+b /(1+c . t)^{d}
$$

where $t$ is related to time, $a+b$ is the curve's initial value ( $\mathrm{t}=0), a$ is its final value $(t=\infty), c$ is related to the decay velocity for small $t$ and $d$ is related to enhancement in velocity decay, more pronounced for great values of $t$.

After all samples had been measured two hundred seeds were taken from each lot and induced to germinate in a controlled chamber $\left(\mathrm{T}=30 \pm 1{ }^{\circ} \mathrm{C}\right.$, humidity $>70 \%$ ) for 30 days. For that, each group of 200 were divided in $4 \times 50$ seeds, and each sub-group disposed in rolls of paper towels moistened with water equivalent to 2 and $1 / 2$ times the dry paper substrate weight. At the 15 th day after start and at the end, at the 30th day, seeds of ' $A$ ' series was analyzed in terms of hypocotyl-radicle axis growth, measuring each seedling elongation; effective germination rate was determined for ' $\mathrm{A}$ ' and ' $\mathrm{B}$ ' series.

\subsection{Results and Discussion}

The DL time profiles of all groups of the first series of tests (Table 9.1) are shown at Fig. 9.2, as well as their correspondent hyperbolic decay fitting $\left(\mathrm{R}^{2}>0.995\right)$ parameters: $a, b, c$ and $d$. It is clear from Fig. 9.2 that groups A7, A11 and A13 present higher initial value than the other groups. Group A17 time profile is also distinguishable from the remaining curves, with small increase from 1 to $100 \mathrm{~ms}$. 
Fig. 9.2 DL data for groups A7 to A18 (left) - 10 test average time profile; (right) parameters of hyperbolic decay fitting $\left(\mathrm{R}^{2}>0.995\right)$
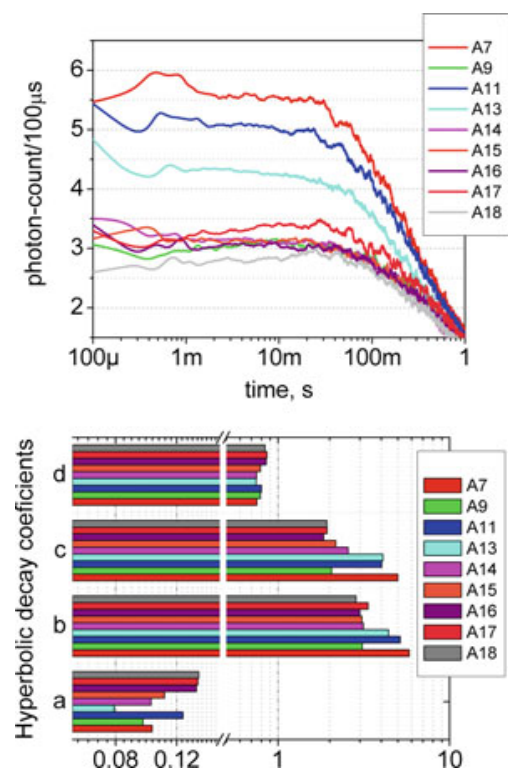

These facts are so reflected in the $b$ parameter of their DL. It is also noted that the $c$ parameter is also higher for the A7, A11 and A13 samples, meaning that their DL intensity decay faster that the other groups, as can be seen also in the time profiles form 10 to $100 \mathrm{~ms}$. The A15 time profile is also noticed to have small increase at the beginning, i.e. $\mathrm{t}<1 \mathrm{~ms}$, as occurring for the curves of A7, A11 and A13.

The remaining groups-A9, A14, A16 and A18 - have similar time profiles, with small initial value $(\sim 3)$ and regular decay velocity, i.e. similar $c$ factor. The germination data - seedling's elongation incidence, in a total of 200 seeds/groupat the 15th and 30th day are presented at Fig. 9.3 for the viable groups; the ones not displayed had no seedling development at all, i.e. zero seeds alive. It is noted that groups A7 and A13 present the higher development of all, followed by A11 and, with much lower development, by A15 and, much lower, A17. The A9 had few seedlings developed.

By summing all seedlings' length the total elongation is obtained for each group, for both the 15th and 30th day after imbibition. This numbers were so plotted against the correspondent $b$ and $c$ parameters, and these datagrams are shown at Fig. 9.4. Good linear correlation was found between the hyperbolic fitting parameters and the total seedling elongation for both the 15th and 30th day data, with $\mathrm{R}^{2}>0.85$ in all cases.

The data obtained for the second, B series of aged seeds (Table 9.2) were analyzed in similar manner as done for the A series, but using just the total germination rate at the 30th day, instead of the hypocotyl-radicle axis growth used before. In this case with most of lots with very good germination, the DL curves 

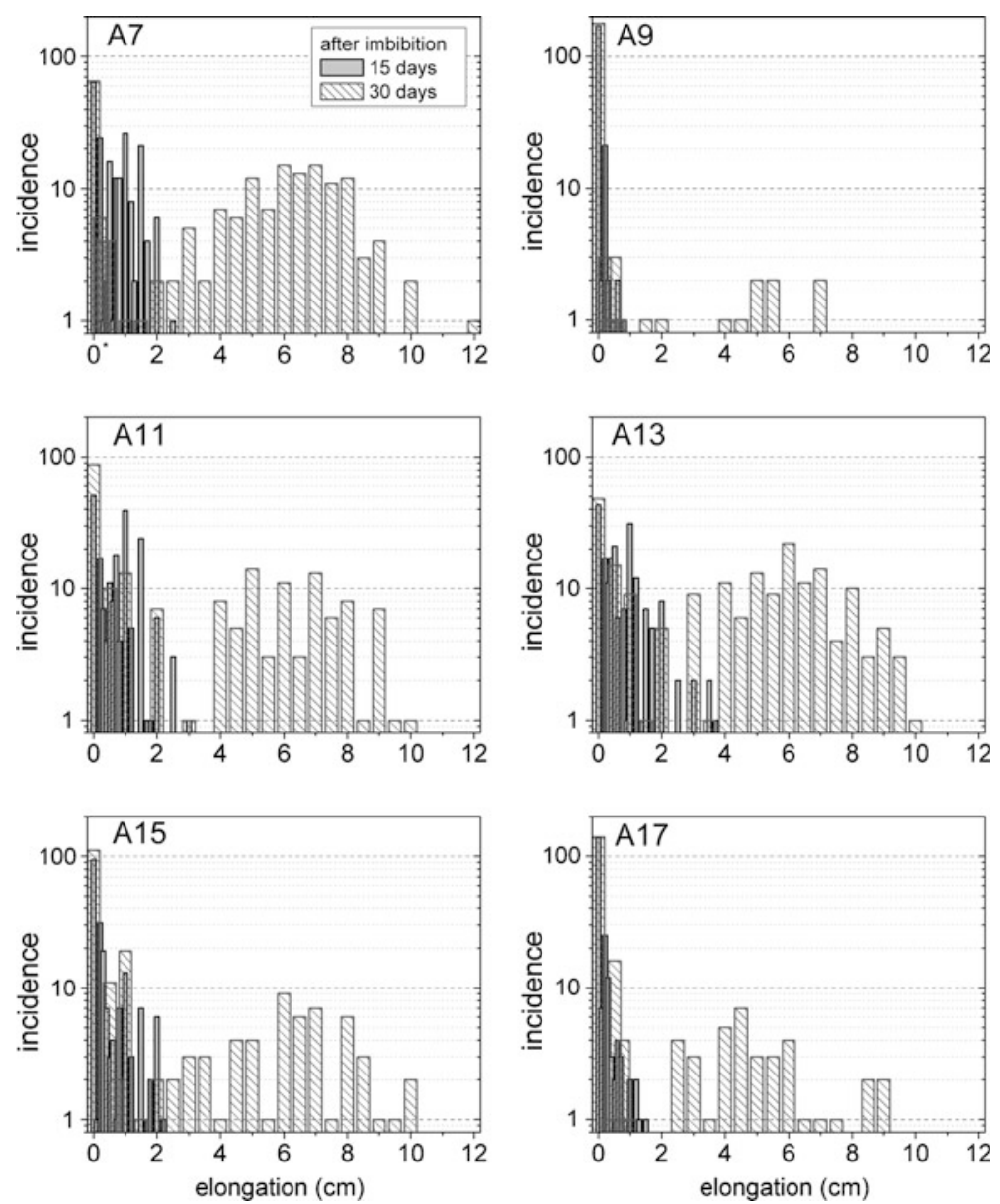

Fig. 9.3 Seedling's length for series A (Table 9.1) for viable groups at the 15th and 30th days after imbibition — each group has 200 seeds total

were not so different for longer $t$, making harder the hyperbolic decay fitting to converge. So, the parameter $d$ was fixed $[d=1,(9.1)]$ to promote a lower standard deviation for the $c$ parameter. The germination rate at the 30th day related to the $b$ and $c$ factors are plotted at Fig. 9.5.

Even after aging, lots of B series presented high germination rate $(>75 \%)$, and very similar DL curves - only exception is sample \#1, with $40 \%$ germination rate. With so, parameters $b$ and $c$ presented much smaller variation than that obtained for the A series. Even though the datagrams of Fig. 9.5 could be fitted by a linear relation $\left(\mathrm{R}^{2}>0.75\right)$. 

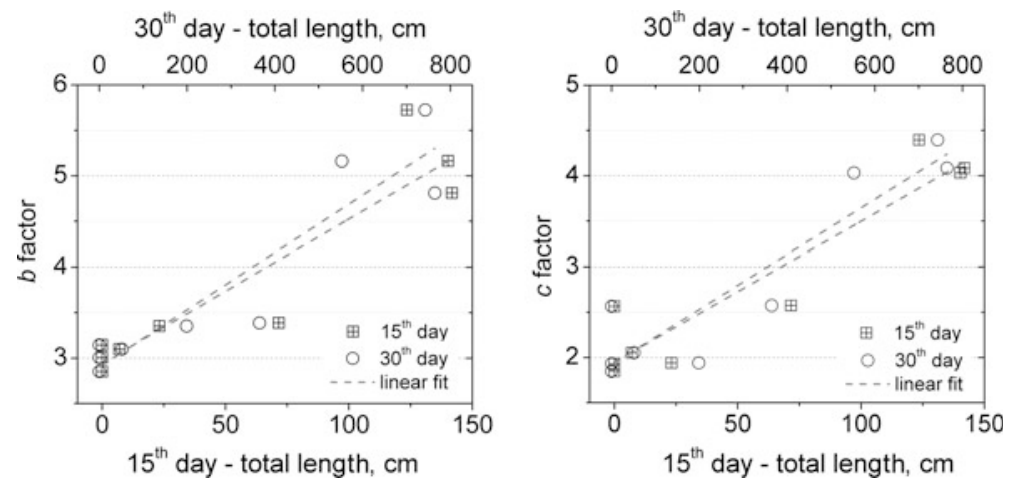

Fig. 9.4 Seedling's total length at the 15th and 30th day versus the hyperbolic decay fitting parameters - (left) the $b$ factor and (right) the $c$ factor; dashed line correspond to linear regression $\left(\mathrm{R}^{2}>0.85\right)$
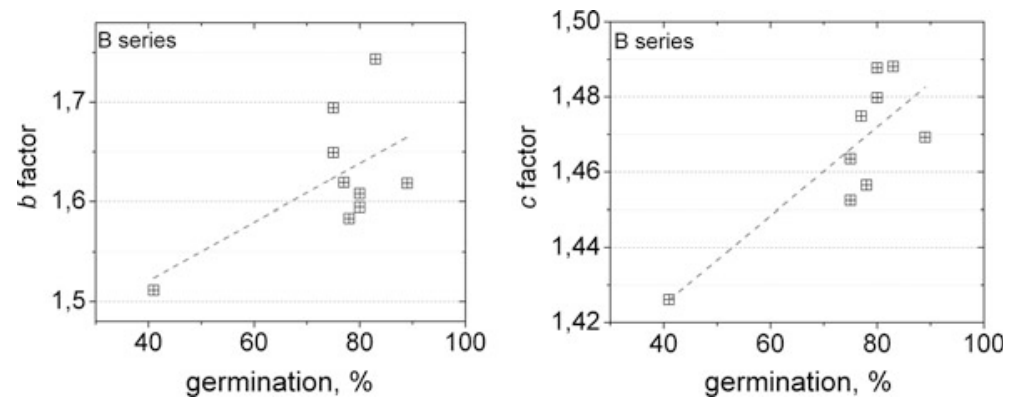

Fig. 9.5 Germination rate at the 30th day versus the hyperbolic decay fitting parameters-(left) the $b$ factor and (right) the $c$ factor $(d=1)$; dashed line correspond to linear regression $\left(\mathrm{R}^{2}>0.75\right)$

\subsection{Conclusions}

The ultra-weak delayed luminescence time profiles of two series of nine groups of coffee seeds with different germination capacity were studied in terms of hyperbolic decay parameters, and good correlations were found between the DL initial value and the decay velocity versus the germinating vigour-i.e., total seedling elongation at the 15th and 30th day after imbibition.

The first, preliminary series 'A' used old samples (2011), already with vigour depreciated by the long storage time in natural conditions, and so just few groups presented significant germination while four of them were almost dead.

The second series 'B', using very fresh samples (2013) with high germination capability even after aging treatment, was less diverse in terms of DL parameters than series 'A', even though a linear relation was found when considering constant the exponential factor $(\mathrm{d}=1)$. 
Although limited in number and range of seed quality, the preliminary data here presented show that the DL measurements of coffee seeds may be used as a fast, non-invasive, non-destructive test to verify sample's viability, and so help in improving post-harvest treatment, storing methods and maybe also beverage quality.

Until now, our preliminary results indicate that DL presents good correlation for low quality seeds. Coffee beans have both embryo and endosperm alive, but the largest proportion is defined by endosperm. As the germination is related to the embryo development more studies are needed improve the knowledge about the seed quality behavior in relation to DL measurements. Next series of experiments would replicate this type of analysis but using a single lot of fresh seed samples ( $<1$ year), which may present high physiological quality, and from which sub-lots would be separated and artificially stressed (aged) in effective manner, in order to have samples with intermediate vigour. With all samples taken from the same lot and underwent different aging treats it is expected to have better view of the relation between germination, vigour and the DL parameters.

Acknowledgements Authors are grateful to the Brazilian Coffee Research Consortium (Embrapa Coffee/UFLA/IAC) for providing the seed samples, to CNPq (Conselho Nacional de Pesquisa), to FAPESP (Fundação de Amparo à Pesquisa do Estado de São Paulo, \#04/10146-3) for partial laboratory support, and to the technical staff supporting LaFA/FT.

\section{References}

1. M.T. Eira, E.A. Silva, R.D. De Castro, S. Dussert, C. Walters, J.D. Bewley, H.W. Hilhorst, Coffee seed physiology. Braz. J. Plant Physiol. 18(1), 149-163 (2006)

2. E. Couturon, Maintaining the viability of coffee seeds by checking their water-content and storage-temperature. Cafe Cacao 24(1), 227-232 (1980)

3. T.D. Hong, R.H. Ellis, Optimum air-dry seed storage environments for arabica coffee. Seed Sci. Technol. 20(3), 547-560 (1992)

4. S. Dussert et al., Benefficial effect of post-having osmoconditioning on the recovery of cryopreserved coffee (Coffea spp.) seeds. Cryo-Lett. 21(1), 47-52 (2000)

5. S. Dussert, N. Chabrillange, G. Rocquelin, F. Engelmann, M. Lopez, S. Hamon, Tolerance of coffee (Coffea spp.) seeds to ultra-low temperature exposure in relation to calorimetric properties of tissue water, lipid composition, and cooling procedure. Physiol. Plant 112(4), 495-504 (2001)

6. M. Kobayashi, H. Inaba, Photon statistics and correlation analysis of ultraweak light originating from living organisms for extraction of biological information. Appl. Opt. 32(1), 183-192 (2000)

7. Michal Cifra, Pavel Pospíšil, Ultra-weak photon emission from biological samples: Definition, mechanisms, properties, detection and applications. J. Photochem. Photobiol. B Biol. (2014). doi:10.1016/j.jphotobiol.2014.02.009

8. Y. Yan, F.A. Popp, G.M. Rothe, Correlation between germination capacity and biophoton emission of barley seeds (Hordeum vulgare L.). Seed Sci. Technol. 31(2), 249-258 (2003) 
9. L. Lanzanò, L. Sui, E. Costanzo, M. Gulino, A. Scordino, S. Tudisco, F. Musumeci, Time-resolved spectral measurements of delayed luminescence from a single soybean seed: effects of thermal damage and correlation with germination performance. Luminescence $\mathbf{2 4}(6)$, 409-415 (2009)

10. Y. Yong, W. Jun, Ultra-weak bioluminescent and vigour of irradiated rice. Int. J. Agric. Biol. Eng. 3(1), 85-90 (2010)

11. J. Wang, Y. Yu, Relationship between ultra-weak bioluminescence and vigour or irradiation dose of irradiated wheat. Luminescence 24(4), 209-212 (2009)

12. C.M. Gallep, E. Conforti, M.T. Braghini, M.P. Maluf, Y. Yan, F.A. Popp, Ultra-weak delayed luminescence in coffee seeds (Coffea arabica and C. canephora) and their germination potential: some indications for a photonic approach in seed viability, in Proceeding of 11th Brazilian Symposium of Microwave and Optoeletronics (2004)

13. E. Bertogna, S.R. dos Santos, J.E.S. Paterniani, E. Conforti, C.M. Gallep, Compact, automatic set-up for ultra-weak photon emission measurements in organisms. SBMO/IEEE MTTS International Microwave and Optoelectronics Conference (IMOC), pp. 449-452. doi:10.1109/ IMOC.2011.6169247 (2011) 